University of Massachusetts Amherst

From the SelectedWorks of Derek Lovley

2002

Analysis of the Genetic Potential and Gene Expression of Microbial Communities Involved in the In Situ Bioremediation of Uranium and Harvesting Electrical Energy from Organic Matter

Derek Lovley, University of Massachusetts - Amherst 


\title{
Analysis of the Genetic Potential and Gene Expression of Microbial Communities Involved in the In Situ Bioremediation of Uranium and Harvesting Electrical Energy from Organic Matter
}

\author{
DEREK R. LOVLEY
}

\begin{abstract}
The proposed research will investigate two microbial communities that are of direct relevance to Department of Energy interests. One is the microbial community associated with the in situ bioremediation of uranium-contaminated groundwater. The second is a microbial community that harvests energy from waste organic matter in the form of electricity. These studies will address DOE needs for (1) remediation of metals and radionuclides at DOE sites and (2) the development of cleaner forms of energy and biomass conversion to energy. Our previous studies have demonstrated that the microbial communities involved in uranium bioremediation and energy harvesting are both dominated by microorganisms in the family Geobacteraceae and that the organisms in this family are responsible for uranium bioremediation and electron transfer to electrodes. The intial objectives of this study are to (1) describe the genetic potential of the Geobacteraceae that predomnate in the environments of interest; (2) identify conserved patterns of gene expression within the Geobacteraceae family in response to a range of environmental conditions; (3) begin to identify mechanisms controlling the expression of key genes related to survival, growth, and activity in subsurface environments and on electrodes; and (4) use the results from subobjectives 1-3 to develop a conceptual model for predicting gene expression of Geobacteraceae in the environments of interest. This will serve as the basis for a subsequent simulation model of the growth and activity of Geobacteraceae in the subsurface and on electrodes.
\end{abstract}

\section{INTRODUCTION}

$\mathrm{T}$ HE PURPOSE OF THIS RESEARCH is to develop conceptual and computational models of the functioning of two microbial communities in environments that are important to needs of the Department of Energy (DOE). These are (1) uranium-contaminated subsurface sediments undergoing in situ bioremediation and (2) the surface of electrodes in which microorganisms are catalyzing the harvesting of energy in the form of electricity from waste organic matter. The study of these environments will benefit DOE in the key ar-

Department of Microbiology, University of Massachusetts, Amherst, Massachusetts. 
eas of (1) remediation of metals and radionuclides at DOE sites and (2) the development of cleaner forms of energy and biomass conversion to energy. The experimental and computational approaches and tools developed during the study of these two environments are likely to have application to the study of the structure and function of microbial communities in other environments of interest to DOE and other federal agencies.

The research team includes the following co-principal investigators: Maddalena Coppi, UMASS; Stacy Ciufo, UMASS; Barbara Methe, The Institute for Genomic Research; Pablo Pomposiello, UMASS; Steve Sandler, UMASS; Cinthia Nunez, UMASS; Daniel Bond, UMASS; Susan Childers, UMASS; Carol Giometti, Argonne National Laboratory; and Julia Krushkal, University of Tennessee.

\section{Analysis and modeling of microbial communities involved in uranium bioremediation}

The microbial communities associated with the in situ bioremediation of uranium contamination in subsurface environments have been chosen for study because the clean-up of environments contaminated with metals and radionuclides is a key need for DOE. Of the metal contaminants at DOE sites, uranium is the most prevalent, both in concentration and in number of contaminated sites. Furthermore, uranium is of particular concern because its generally high solubility, and hence mobility in groundwater, leads to the spread of uranium and the potential for contamination of important water resources.

One of the most promising strategies for preventing the spread of subsurface uranium contamination is microbial uranium reduction (Lovley et al., 1991; Lovley, 1995; Lovley and Anderson, 2000; Anderson and Lovley, 2002; Finneran et al., 2002a). The uranium in uranium-contaminated groundwaters is in the U(VI) form, which is highly soluble in most natural waters. Under the appropriate conditions dissimilatory metalreducing microorganisms use $\mathrm{U}(\mathrm{VI})$ as a terminal electron acceptor. The highly soluble $\mathrm{U}(\mathrm{VI})$ is reduced to U(IV), which is highly insoluble and precipitates from the groundwater as the mineral uraninite. Thus, microbial U(VI) reduction can effectively remove U(VI) from contaminated water and prevent further migration of uranium contamination in the subsurface. In contrast to the highly effective biological reduction of U(VI) with dissimilatory metal-reducing microorganisms, it is difficult, if not impossible, to chemically reduce $U(V I)$ with reducing agents under conditions found in subsurface environments.

A wide phylogenetic diversity of microorganisms are known to reduce U(VI) and other metals in pure culture (Lovley, 2000b). In order to better understand the process of U(VI) reduction in subsurface environments, molecular analyses have been conducted in a wide variety of subsurface environments in which microbial metal reduction is an important process in situ. These studies have invariably found that a single family of microorganisms predominates in subsurface environments under the environmental conditions most representative of those found at the majority of DOE sites. These are microorganisms in the family Geobacteraceae.

The predominance of Geobacteraceae under metal-reducing conditions in subsurface environments was first observed in petroleum-contaminated aquifer sediments in which microbial Fe(III) reduction was an important process for removing hydrocarbon contaminants from the groundwater (Rooney-Varga et al., 1999). Subsequent field studies in which petroleum or other organic compounds were added to the subsurface to promote Fe(III) reduction also resulted in an enrichment of Geobacteraceae (Snoeyenbos-West et al., 2000). Molecular analysis of subsurface sediments contaminated with land-fill leachate (Röling et al., 2001) as well as aquatic sediments in which metal reduction was important (Stein et al., 2001) also found that Geobacteraceae were the most prevalent microorganisms.

Most relevant to DOE interests were the results from several uranium mine tailings (UMTRA) sites (Finneran et al., 2002b). Prior to treatment, these organic-poor aquifers were aerobic. Uranium was in the $\mathrm{U}(\mathrm{VI})$ state and thus mobile. When acetate or other electron donors were added to the sediments anaerobic respiration was stimulated. Once any nitrate present was reduced, Fe(III) and U(VI) reduction proceeded concurrently. U(VI) was rapidly and effectively removed from the groundwater. There was no sulfate reduction during the $\mathrm{U}(\mathrm{VI})$ reduction, which suggested that sulfate-reducing microorganisms were not involved in U(VI) reduction. Simultaneous with the onset of Fe(III) and U(VI) reduction was a significant increase in Geobacteraceae in the sediments. The numbers of Geobacteraceae increased from being barely detectable in the aerobic sediments prior to treatment to accounting for over $40 \%$ of the microbial com- 


\section{MICROBIAL COMMUNITIES AND THE IN SITU BIOREMEDIATION OF URANIUM}

munity during the Fe(III) and U(VI) reduction phase (Holmes et al., 2002b). Such an enrichment of a single group of microorganisms in a soil sample is unprecedented. There was no apparent increase in any other group of microorganisms. The fact that the increase in Geobacteraceae was associated with the initiation of $\mathrm{Fe}(\mathrm{III})$ and $\mathrm{U}(\mathrm{VI})$ reduction and the fact that microorganisms in this family are known to grow via Fe(III) and U(VI) reduction (Lovley, 2000a; Lovley, 2000b) indicate that the Geobacteraceae were responsible for the metal reduction in the uranium-contaminated sediments (Holmes et al., 2002b).

These results suggest that, in order to understand the factors controlling the rate and extent of U(VI) reduction in subsurface environments and to rationally design strategies to optimize in situ uranium bioremediation, it is important to understand the physiology and metabolism of the Geobacteraceae that are responsible for metal reduction in these environments. Information is required not only on how these microorganisms reduce U(VI), but also on how growth is coupled to Fe(III) reduction, because even in subsurface environments that are heavily contaminated with uranium, Fe(III) accounts for over $95 \%$ of the electron-accepting capacity supporting the growth of the Geobacteraceae (Finneran et al., 2002a). Many other unknown physiological factors also require investigation, such as how do Geobacteraceae survive the oxygen stress that they face in aerobic subsurface environments prior to the stimulation of anoxic conditions and how do they survive in such nutrient-poor environments. Although some information that can help answer these questions is likely to come from the study of the Geobacteraceae that have been isolated in pure cultures from other sites, more accurate information is likely to be obtained from the study of the Geobacteraceae populations that predominate in uranium-contaminated subsurface sediments. Therefore, a major goal of the research proposed here is to evaluate the genetic potential and gene expression of the Geobacteraceae involved in uranium bioremediation in subsurface environments in order to be able to predictively model their activity under different environmental conditions that naturally exist or might be artificially imposed in an engineered bioremediation of uranium-contaminated subsurface environments.

\section{Analysis and modeling of microbial communities involved in harvesting electricity from organic matter}

Another goal of DOE is to promote the development of cleaner forms of energy and the conversion of biomass to useable energy forms. We have recently discovered a novel form of microbial metabolism which shows great promise for converting waste organic matter to electricity (Bond et al., 2002; Holmes et al., 2002a). We demonstrated for the first time that there are microorganisms that can effectively transfer electrons that are derived from the oxidation of organic matter onto the surface of electrodes. This represents a clean, non-polluting strategy for converting organic matter of little or no economic value into a highly desirable form of energy. Surprisingly, the microorganisms that are responsible for this energy conversion are also the Geobacteraceae.

Earlier studies had demonstrated that if a graphite electrode is buried in anaerobic marine sediments and connected with a wire to a graphite electrode in the overlying aerobic water, then there will be a flow of current (Reimers et al., 2001). In these early studies it was assumed that the electrode in the sediment (the anode) was accepting electrons from reduced products of microbial metabolism such as sulfide, ferrous iron, and reduced humic substances (Reimers et al., 2001). However, subsequent studies have suggested that these reduced products account for, at best, only a minor portion of the current output (Tender et al., 2002).

We examined the surface of the sediment anodes to determine if there might be a specialized community associated with the anodes that might account for the current production. In examining anodes from laboratory and field experiments, conducted with sediments from a variety of marine and freshwater environments, we found that the energy-harvesting anodes were always highly enriched in microorganisms in the Geobacteraceae family, which accounted for approximately half of all the microorganisms colonizing the anodes in sediments (Bond et al., 2002; Holmes et al., 2002a). Studies with pure cultures of a variety of Geobacteraceae demonstrated that they all had the ability to oxidize organic compounds to carbon dioxide with the electrode serving as the sole electron acceptor. The stoichiometry of organic matter oxidation and electricity production indicated that the electrons derived from organic matter oxidation were being quantitatively transferred to the electrode. This is the first description of this ability in microorganisms. Although other types of microbial fuel cells have been studied previously, in each instance those fuel cells 
required the addition of an exogenous electron-shuttling mediator to promote effective electron transfer between the microorganisms and the electrode. The Geobacteraceae do not require an electron shuttle and can directly transfer electrons to the electrode. This direct electron transfer makes it possible to harvest energy from a wide variety of environments and types of organic matter without the concern about the use of expensive and toxic mediator compounds.

The Geobacteraceae can use a wide variety of organic compounds as electron donors for electricity production. These include acetate, the central extracellular intermediate in the anaerobic degradation of organic matter. This demonstrates that complex organic matter can be converted to electricity with Geobacteraceae and suggests that microorganisms should be able to produce electricity from waste organic matter such as sewage sludge and from a variety of forms of biomass. Furthermore, organic contaminants such as toluene could be anaerobically oxidized with the electrode serving as the sole electron acceptor, suggesting that electrodes may be useful for stimulating the microbial degradation of organic contaminants in subsurface environments and sediments.

Much more needs to be learned about functioning of the microbial community on the electrodes in order to optimize this novel form of energy harvesting. The significant enrichment of Geobacteraceae on energy-harvesting electrodes clearly indicates that these organisms have a competitive advantage in using electrodes as an electron acceptor. However, nothing is known about the genetic potential and physiological state of the Geobacteraceae community living on the sediment anodes. Such information is crucial to further developing this process.

\section{Geobacteraceae-known physiology and ongoing functional genomics and modeling studies}

Valuable information that will provide some insights into the functioning of the Geobacteraceae that are involved in subsurface uranium bioremediation and harvesting energy from waste organic matter can be obtained from the study of pure cultures of these organisms. Although detailed studies on the physiology and biochemistry of Geobacteraceae has only recently begun in earnest, progress is being made rapidly due to (1) the availability of extensive genomic information on several Geobacteraceae species; (2) the recent development of a genetic system for these organisms (Coppi et al., 2001); (3) the development of methods for mass culturing these organisms as well the ability to grow them in chemostats; (4) ongoing proteomic studies; and (5) the availability of a whole genome DNA microarray for studying gene expression in Geobacter sulfurreducens.

Recent studies, which are funded under the DOE Microbial Genome Program, have primarily focused on G. sulfurreducens. The complete genome for this organism is available (geobacter.org) and detailed functional genomic studies involving classical genetic and biochemical approaches as well as gene expression studies using the whole genome DNA microarray and detailed proteomic profiling are beginning to elucidate the function of many of the genes in this organism. Partial genome sequences for other species with the Geobacteraceae including Geobacter metallireducens, Desulfuromonas acetoxidans, and Pelobacter carbinolicus have been provided by the DOE Joint Genome Institute and we plan to complete those genomes at UMASS over the next year. Bernhard Palsson, Christophe Schilling, and colleagues at Genomatica (www.genomatica.com) are producing a preliminary computer model of G. sulfurreducens metabolism, based upon the whole genome sequence and this model will be further developed as more functional genomic data becomes available.

Within the last year our understanding of the mechanisms by which Geobacter species access and reduce insoluble $\mathrm{Fe}$ (III) oxides has increased dramatically. Understanding electron transfer to Fe(III) is important because $\mathrm{Fe}$ (III) is the primary electron acceptor supporting the growth of Geobacteraceae, even in uranium-contaminated environments. Differential expression studies indicated that several $c$-type cytochromes were produced to a much greater extent when Fe(III) served as the electron acceptor. Knockout mutations in the genes for these cytochromes permitted evaluation of the role of these cytochromes in electron transport to $\mathrm{Fe}(\mathrm{III})$. This has led to a new model for electron transport to $\mathrm{Fe}(\mathrm{III})$ oxides.

Another early finding in our functional genomics studies is the discovery of a novel mechanism for Geobacter species to access Fe(III) oxides. Earlier studies had demonstrated that, unlike other Fe(III)-reducing microorganisms such as Shewanella and Geothrix species, the Geobacter species do not produce 
electron shuttling compounds or Fe(III) chelators that might alleviate the need for Geobacter to directly contact Fe(III) oxide in order to reduce it (Nevin and Lovley 2002b). This led to the question of how Geobacter could find the Fe(III) oxides that serve as its primary electron acceptor in subsurface environments, because Geobacter species had been described as non-motile. However, inspection of the preliminary genome sequence data indicated that Geobacter species had genes for flagella, suggesting that they might be motile under some conditions. Further investigation revealed that they were motile, but only when growing with insoluble Fe(III) or Mn(IV) oxides as the electron acceptor (Childers et al., 2002). Examination with electron microscopy revealed that flagella and pili were produced during growth on the insoluble oxides, but not during growth on soluble electron acceptors, including chelated Fe(III) forms. The genes for pili production were identified in the genome and gene expression studies demonstrated that genes for pili were only expressed during growth on $\mathrm{Fe}(\mathrm{III})$ and $\mathrm{Mn}$ (IV) oxides. Further examination of the genome revealed the presence of genes for chemotaxis and chemotaxis studies demonstrated that G. metallireducens was chemotactic to the $\mathrm{Fe}$ (II) and $\mathrm{Mn}$ (II) that emanate from $\mathrm{Fe}(\mathrm{III})$ and $\mathrm{Mn}$ (IV) oxides under anoxic conditions (Childers et al., 2002). Knockout mutations in pilA, the gene for the structural pilin protein, and pilT, the gene for twitching motility demonstrated that Geobacter required pili in order to reduce insoluble Fe(III) oxide and used the pili not only to attach to Fe(III) oxide, but also to move along sand grains in order to localize Fe(III) oxide coatings (T. Mehta, unpublished data). These studies have revealed that Geobacter species have a very energy efficient strategy for accessing Fe(III) oxides in order to reduce them. Other well-studied Fe(III)-reducing microorganisms such as Shewanella and Geothrix species, appear to lack this mechanism for accessing Fe(III) oxides and produce energetically expensive electron-shuttling compounds and $\mathrm{Fe}$ (III) chelators. It is likely that this difference in the mechanisms for accessing Fe(III) oxides explains, at least in part, why Geobacteraceae are the predominant Fe(III)-reducing microorganisms in a variety of sedimentary environments.

Thus, it is apparent that functional genomics studies are beginning to reveal some of the key mechanisms that permit Geobacteraceae to compete and thrive in subsurface environments. However, there are many other aspects to the physiology of these organisms that are important for their survival and activity in the subsurface that have yet to be elucidated. Furthermore, there is almost no information on factors influencing growth and electron transfer of Geobacteraceae living on electrodes. Finally, although we have some preliminary information on the genetic potential of some Geobacteraceae available in pure culture, there is little information on the genetic potential of the "as-yet-uncultured" Geobacteraceae living in environments in which they catalyze environmentally significant processes.

\section{MATERIALS AND METHODS}

\section{Study sites and microbial communities to be investigated}

The two environments of interest are uranium-contaminated subsurface sediments undergoing uranium bioremediation and the surface of electrodes harvesting energy from organic matter. The Old Rifle UMTRA site in Colorado is serving as the uranium-contaminated subsurface environment for these studies. This is the site of an ongoing uranium bioremediation field experiment. The environmental genomics studies described here will be supported by detailed geochemical and microbiological characterization of the site. The electrode studies will be conducted with electrodes emplaced in organic-rich freshwater sediments and electrodes emplaced in laboratory reactors fed sewage sludge. Organic-rich freshwater sediments, such as those found in constructed wetlands for treating wastewaters represent a large source of potential energy, but there is no current means for harvesting this energy in a useable form. Although attempts have been made to harvest energy from sewage sludge in the form of methane, by using methanogenic treatment systems, such methanogenic systems are difficult to operate and have not met with wide application. Preliminary studies have demonstrated that just as in marine environments, Geobacteraceae also predominate on the surface of electrodes harvesting energy from freshwater environments (D. Holmes, unpublished data).

The first three years of these studies will focus solely on the Geobacteraceae that predominate in the uranium-contaminated subsurface sediments undergoing bioremediation and on energy-harvesting electrodes. Ideally, it is desirable to have information on each microorganism in a microbial community. How- 
ever, our preliminary results have demonstrated that Geobacteraceae account for nearly half of the microbial communities that will be investigated, the other half of the community is comprised of a wide diversity of microorganisms, none of them accounting for a significant fraction of the community. It is probably not an effective use of time or money to obtain large amounts data from this wide diversity of non-Geobacteraceae because they do not appear to be important in the processes of interest. In the case of the uranium-contaminated sediments, the non-Geobacteraceae do not increase in numbers during uranium bioremediation and appear to be relics of the pre-treatment phase. In the case of the electrodes, the nonGeobacteraceae on the energy-harvesting electrodes are similar to those found on control electrodes emplaced in the sediments, but not electrically connected to an anode, suggesting that they are non-specifically attached to the electrode surface. In contrast to the opportunity to conduct defined, coherent studies on the Geobacteraceae in the environments of interest, data on the non-Geobacteraceae in these environments is likely to be largely uninterpretable and is not likely to provide significant insights into the functioning of these communities. Furthermore, considerable effort would be required to study each of these minor organisms and each intensive study would only provide information on a minor portion of the microbial community. In contrast, by focusing on the Geobacteraceae component of the microbial community it is possible to leverage the intensive ongoing functional genomic and modeling studies on pure cultures of Geobacteraceae while gaining detailed information on the genetic potential and functioning of this predominant segment of the microbial community. If the studies described here are successful, then if warranted, it will be possible to conduct studies on the more minor components of the microbial communities in the future.

\section{Objectives and hypotheses}

The ultimate objective of these studies is to develop models that can predict the activity of Geobacteraceae-dominated microbial communities in uranium-contaminated subsurface environments and on energyharvesting electrodes in order to predict the success of bioremediation and energy harvesting under different environmental conditions; and aid in the optimization of these strategies. The subobjectives in the first three years of this study are to (1) describe the genetic potential of the Geobacteraceae that predominate in subsurface sediments undergoing uranium bioremediation and on energy-harvesting electrodes; (2) identify conserved patterns of gene expression within the Geobacteraceae family, including the Geobacteraceae living in the environments of interest, in response to a range of environmental conditions; (3) begin to identify mechanisms controlling the expression of key genes related to survival, growth, and activity in subsurface environments and on electrodes; and (4) use the results from subobjectives 1-3 to begin developing a conceptual model for predicting the growth and activity of Geobacteraceae in the environments of interest. This will provide the basis for a simulation model of the growth and activity of Geobacteraceae in the subsurface and on electrodes that will be developed in the future.

\section{Subproject I: genetic potential of Geobacteraceae in uranium-contaminated subsurface environments and on the surface of energy-harvesting electrodes}

The objectives will be addressed in three subprojects. The first subproject is designed to better understand the genetic potential of Geobacteraceae in the environments of interest. It is hypothesized that it is possible to obtain complete, or nearly complete, genome sequences of the individual Geobacteraceae species that predominate in subsurface environments undergoing uranium bioremediation and on the surface of energy-harvesting electrodes. One strategy for recovering these genome sequences will be to recover, in pure culture, a large proportion of the environmentally predominant Geobacteraceae from uranium-contaminated subsurface sediments undergoing bioremediation and from the surface of energy-harvesting electrodes. Sequencing the genomes of these organisms can provide the complete genome sequences of these Geobacteraceae in the environments of interest.

We have developed methods for culturing a diversity of Geobacteraceae from a variety of natural environments. The ability to obtain Geobacteraceae in culture contrasts with the general finding in environmental microbiology that the microorganisms responsible for environmental processes of interest can not 
be recovered in pure culture. Based upon our past studies in other environments, it can be expected that it will be possible to culture at least some of the Geobacteraceae that predominate in the two environments of interest. Once these organisms are in culture it will be a simple matter to sequence their complete genomes using standard techniques. In this manner it will be possible to obtain complete genomes of at least some of the Geobacteraceae that predominate in the environments of interest.

However, it is expected that the isolation procedures will only recover a portion of the Geobacteraceae that predominate in the environments of interest. We hypothesize that it will be possible to sequence the genomic DNA of predominant but "not-yet cultured" Geobacteraceae and assemble these sequences into large contigs that represent significant portions of the genomes of the "not-yet -cultured" Geobacteraceae. We have already demonstrated that we can readily recover large segments of Geobacteraceae genomic DNA from environmental samples. Furthermore, we have used UMASS-developed software, known as Bacpack, to perform in silico experiments that demonstrate that it should be possible to assemble this genomic DNA into large contigs that can be assigned to individual strains of "not-yet-uncultured" Geobacteraceae. New computational tools for analyzing environmental genomic sequences are also under development.

We further hypothesize that analysis of the genomes of the Geobacteraceae predominating in uraniumcontaminated subsurface sediments undergoing bioremediation and on energy-harvesting electrodes will reveal that much of the genetic potential of the Geobacteraceae living in the environments of interest will be shared with other, more of the intensively studied, Geobacteraceae that are available in pure culture. It is expected that, within the Geobacteraceae, there is significant conservation of genetic potential for the primary functions related to growth and survival in the environments of interest. In order to evaluate this it will be necessary to analyze the genomes of organisms that represent the phylogenetic and physiological range of the Geobacteraceae. Therefore, in addition to continuing our in-depth characterization of the genome of Geobacter sulfurreducens we propose to finish the genomes of the partially sequenced Geobacter metallireducens and Desulfuromonas acetoxidans and then completely sequence the genomes of Pelobacter carbinolicus and "Desulfuromusa electrodophilus," a recent electrode isolate. The genomes of these organisms, as well as those of any of the predominant Geobacteraceae that are isolated from the environments of interest, will be analyzed to provide the detailed data that is required in order to adequately model the genetic potential of the Geobacteraceae. The same computational tools used to evaluate similarities and differences in the genetic potential of existing pure cultures will be used to assess the genetic potential of the "not-yet-uncultured" Geobacteraceae through an analysis of the environmental genomic Geobacteraceae sequences.

\section{Subproject II: gene expression of Geobacteraceae in uranium-contaminated subsurface environments and on the surface of energy-harvesting electrodes}

In order to model the activity of Geobacteraceae in uranium-contaminated subsurface sediments and on the surface of energy-harvesting electrodes, it is necessary to have data on gene expression under relevant environmental conditions. Detailed studies on gene expression in G. sulfurreducens under a variety of environmental conditions are already underway as part of our studies under the Microbial Genome Program. We propose to apply the same whole-genome DNA microarray and proteomics approaches that are being employed in the study of G. sulfurreducens to study the gene expression of the other Geobacteraceae that are available in pure culture to find commonalties in gene expression patterns that will be helpful in developing models for gene regulation and the growth and activity of Geobacteraceae in the environments of interest.

Furthermore, we have modified the DNA-microarray and proteomic approaches that we are using for the study of gene expression in pure cultures to the study of levels of Geobacteraceae mRNA and proteins in subsurface sediments. This will permit the analysis of gene expression within the environments of interest. Studies will be conducted on samples taken directly from the environments of interest as well as on environmental samples incubated in the lab and subjected to a variety of environmental perturbations that are of relevance to uranium bioremediation or energy harvesting. This data, coupled with the pure culture data described above, will indicate whether models of gene expression developed from pure cultures can be used to describe natural microbial communities. 


\section{Subproject III: models for regulation of gene expression in uranium-contaminated subsurface environments and on the surface of energy-harvesting electrodes}

An analysis of the regulatory networks in pure cultures of Geobacteraceae should help to provide an initial framework for describing the patterns of gene expression observed in Geobacteraceae communities in response to various environmental conditions. Regulatory systems in the Geobacteraceae will be identified with a combination of bioinformatic and experimental approaches. The initial emphasis will be on regulatory systems that are likely to be involved in response to important environmental stimuli that may function in a manner similar to that previously described in other microorganisms. For example, some of the global transcription factors that Geobacteraceae may have in common with a variety of other microorganisms will be investigated. These include (1) sigma factors, (2) FUR, and (3) FNR. Two-component regulatory systems, which appear to be abundant in Geobacteraceae, will also be studied in detail and mechanisms regulating the responses to environmental stresses such as oxidative stress, exposure to toxic metals, or nutrient deprivation will be investigated.

\section{CONCLUSION}

This project will provide significant new information on the function of the microbial communities involved in two processes of interest to the Department of Energy, the in situ bioremediation of uranium in subsurface environments and harvesting of electricity from waste organic matter. The study of the function of these communities is simplified by the fact that in both environments, microorganisms in the family Geobacteraceae comprise nearly half of the microbial community. Another advantage in studying these communities is that it is possible to culture Geobacteraceae. Thus, it may be possible to obtain significant information on the genetic potential of the predominant Geobacteraceae in the environments of interest by recovering these organisms in culture and sequencing their genomes. Furthermore, preliminary studies have demonstrated that it is possible to recover large segments of Geobacteraceae genomic DNA directly from sedimentary environments and in silico experiments have indicated that it will be possible to assemble this genomic DNA into large contigs that can be assigned to individual strains of Geobacteraceae. Gene and protein expression of a diversity of Geobacteraceae available in culture, including those recovered from the environments of interest, will be analyzed under various environmentally relevant growth conditions to elucidate gene expression patterns that can be expected in these environments. Furthermore, DNA-microarray and proteomic analyses will also be conducted directly on environmental samples. Studies on mechanisms for gene regulation that employ a combination of bioinformatic and experimental approaches will provide a conceptual framework for explaining and predicting patterns of gene expression. In addition to leading to the development of an in-depth understanding of the microbial communities involved in uranium bioremediation and energy harvesting, another benefit of this research will be the development of experimental and computational tools that will be applicable to the study of other microbial communities.

The conceptual models of genetic potential, gene expression, and regulation that will be the products of the first three years of research will serve as the basis for the development of computational models that can describe the functioning of the Geobacteraceae in the environments of interest. The model for the functioning of the Geobacteraceae community will then be coupled with hydrological and geochemical models to provide the ability to predict the success of in situ uranium bioremediation or energy harvesting under various conditions and to optimize these processes.

\section{REFERENCES}

ANDERSON, R.T., and LOVLEY, D.R. (2002). Microbial redox interactions with uranium: an environmental perspective. Interactions of Microorganisms with Radionuclides. M.J. Keith-Roach and F.R. Livens. Amsterdam, Elsevier Science Limited: 205-223.

BOND, D.R., HOLMES, D.E., TENDER, L.M., et al. (2002). Electrode-reducing microorganisms harvesting energy from marine sediments. Science 295, 483-485. 


\section{MICROBIAL COMMUNITIES AND THE IN SITU BIOREMEDIATION OF URANIUM}

CHILDERS, S.E., CIUFO, S., and LOVLEY, D.R. (2002). Geobacter metallireducens access Fe(III) oxide by chemotaxis. Nature 416, 767-769.

COPPI, M., LEANG, C., LOVLEY, D., et al. (2001). Development of a genetic system for Geobacter sulfurreducens. Appl Environ Microbiol 67, 3180-3187.

FINNERAN, K., ANDERSON, R.T., NEVIN, K.P., et al. (2002a). Bioremediation of uranium-contaminated aquifers with microbial U(VI) reduction. Soil Sediment Contamination 11, 339-357.

FINNERAN, K.T., HOUSEWRIGHT, M.R., and LOVLEY, D.R. (2002b). Multiple influences of nitrate on uranium solubility during bioremediation of uranium-contaminated subsurface sediments. Environ Microbiol 4, 510-516.

HOLMES, D., BOND, D., TENDER, L.M., et al. (2002a). Microbial communities associated with electron-accepting and electron-donating electrodes in sediment fuel cells. Appl Environ Microbiol (manuscript submitted).

HOLMES, D.E., FINNERAN, K.T., and LOVLEY, D.R. (2002b). Enrichment of Geobacteraceae associated with stimulation of dissimilatory metal reduction in uranium-contaminated aquifer sediments. Appl Environ Microbiol 68, 2300-2306.

LOVLEY, D.R. (1995). Bioremediation of organic and metal contaminants with dissimilatory metal reduction. J Industr Microbiol 14, 85-93.

LOVLEY, D.R. (2000a). Fe(III) and Mn(IV) reduction. EnvironmentalMicrobe-Metal Interactions. D.R. Lovley. Washington, DC, ASM Press: 3-30.

LOVLEY, D.R. (2000b). Fe(III)- and Mn(IV)-reducing prokaryotes The Prokaryotes. M. Dworkin, S. Falkow, E. Rosenberg, K.-H. Schleifer and E. Stackebrandt. New York, Springer-Verlag, Inc.: www.prokaryotes.com.

LOVLEY, D.R., and ANDERSON, R.T. (2000). The influence of dissimilatory metal reduction on the fate of organic and metal contaminants in the subsurface. J Hydrol 8, 77-88.

LOVLEY, D.R., PHILLIPS, E.J.P., GORBY, Y.A., et al. (1991). Microbial reduction of uranium. Nature 350, $413-416$.

NEVIN, K.P., and LOVLEY, D.R. (2002a). Mechanisms for accessing insoluble Fe(III) oxide during dissimilatory $\mathrm{Fe}(\mathrm{III})$ reduction by Geothrix fermentans. Appl Environ Microbiol 68, 2294-2299.

NEVIN, K.P., and LOVLEY, D.R. (2002b). Mechanisms for Fe(III) oxide reduction in sedimentary environments. Geomicrobiol J 19, 141-159.

REIMERS, C.E., TENDER, L.M., FERTIG, S., et al. (2001). Harvesting energy from the marine sediment-water interface. Environ Sci Technol 35, 192-195.

RÖLING, W.F.M., VAN BREUKELEN, B.M., BRASTER, M., et al. (2001). Relationships between microbial community structure and hydrochemistry in a landfill leachate-polluted aquifer. Appl Environ Microbiol 67, 4619-4629.

ROONEY-VARGA, J.N., ANDERSON, R.T., FRAGA, J.L., et al. (1999). Microbial communities associated with anaerobic benzene mineralization in a petroleum-contaminated aquifer. Appl Environ Microbiol 65, 3056-3063.

SNOEYENBOS-WEST, O.L., NEVIN, K.P., and LOVLEY, D.R. (2000). Stimulation of dissimilatory Fe(III) reduction results in a predominance of Geobacter species in a variety of sandy aquifers. Microb Ecol 39, $153-167$.

STEIN, L.Y., LA DUC, M.T., GRUNDL, T.J., et al. (2001). Bacterial and archaeal populations associated with freshwater ferromanganous micronodules and sediments. Environ Microbiol 3, 10-18.

TENDER, L.M., REIMERS, C.E., STECHER, H.A., et al. (2002). Harnessing microbially generated power on the seafloor. Nat Biotechnol 20, 821-825.

Addres reprint requests to: Dr. Derek R. Lovley Department of Microbiology University of Massachusetts Amherst, MA 01003

E-mail: dlovley@microbio.umass.edu 